\title{
Invariance of the weight parameter in information integration
}

\author{
IRWIN P. LEVIN, KYUNG JA KIM, and FERNANDO A. CORRY \\ University of Iowa, Iowa City, Iowa 52242
}

\begin{abstract}
The relative effect of each informational dimension in an information integration task is a joint function of its weight and the range of values over which it is varied. A method is developed for separating these two factors. Weights obtained by this method were compared across variations of stimulus range. Subjects rated the performance of students on the basis of midterm exam scores and final exam scores. For some subjects, the range of scores on the final exam was twice that on the midterm and the reverse was true for other subjects. An averaging model was shown to describe the results, and weights did not differ for different stimulus ranges. This was true for each of two instructional conditions: one in which a particular weighting strategy was prescribed and one in which there was no prescribed weighting. Students who were instructed to use a $2: 1$ weighting were found to assign more than twice as much weight to the final as to the midterm.
\end{abstract}

Researchers are often interested in assessing the relative contribution of each of several variables on a particular measure of behavior. An assessment can sometimes be made in a simple manner - e.g., comparing the relative proportion of the variance contributed by each variable. However, the usefulness of such comparisons may be questioned (Dooling \& Danks, 1975). A major problem is that the contribution of a given variable is a joint function of the importance or weight of that variable and the range of values over which it is varied. Even a potentially important variable will have little relative effect if its range is narrowly restricted. For many purposes it would be useful to separate the weighting effect from the range effect.

This paper will examine the problem of defining the importance of a variable apart from the range over which it is varied in an information integration task - i.e., a task where subjects must combine several pieces of information to arrive at a single judgmental response. Anderson's (1974) information integration theory formalizes the problem by assuming that each piece of information in an integration task has two components - a scale value $s$ representing the subjective evaluation of the informational stimulus along the dimension of judgment, and a weight $w$ representing the degree of psychological importance of that piece of information. The net effect of the presentation of a given piece of information is the product $w \mathrm{~s}$. While the values of $\mathrm{w}$ and s cannot generally be separated, Levin (1975, Experiment 2) showed how this could be accomplished in a task where subjects were required to make judgments and ratings based on numerical information. This work was part of a series of studies of numerical information integration (Levin, 1974a, b; 1975), showing that the numbers themselves may be taken as interval scale estimates of their subjective scale values. Since scale values can be obtained directly, weights can be estimated in a straightforward manner.
In the initial study used to illustrate this method (Levin, 1975, Experiment 2), the range of values was equalized for each of two variables and their weights were compared. If the weighting of different stimulus dimensions is to have psychological meaning, weights must be shown to be invariant across variations in stimulus range. This is the primary goal of the present study.

Subjects were asked to rate the performance of each member of a hypothetical class of students on the basis of final exam scores and midterm exam scores. This task has the desirable property that the two stimulus dimensions vary along the same nominal scale. For some subjects, the range of scores on the final exam was twice the range of scores on the midterm, and for some subjects, the range of scores on the midterm was twice the range of scores on the final. Weights were estimated in the context of a simple additive model of information integration and the relative weights of the two types of scores were compared in each case.

In addition to the usual instructional condition where subjects were allowed to choose their own weighting strategy, a unique instructional condition was included where subjects were told to weight the score on the final exam twice as much as the score on the midterm exam. This allowed an assessment of how accurately subjects can produce a prescribed weighting function in making subjective evaluations. It also allowed a second test of weight invariance.

\section{METHOD}

\section{Within-subject manipulations}

In rating the performance of each hypothetical student, subjects were usually given both a final exam score (F) and a midterm exam score (M), but sometirres only one of these scores was given. Each score was presented as percent correct responses. For the trials of interest there were five levels of each type of score. Each subject received two replications of the following 
items: 25 score-pairs formed by combining each level of $F$ with each level of $\mathrm{M} ; 10$ single scores consisting of each level of $F$ and each level of $M ; 14$ filler items, consisting of score-pairs and single scores with values other than those in the main design. Scores more extreme than those in the main design were included in the fillers in order to anchor the ends of the rating response scale. A different random order of presentation was used for each replication.

\section{Between-subjects manipulations}

There were four experimental groups formed by combining two levels of the relative range of $F$ scores and $M$ scores with two types of instructions. For Groups 1 and $3(n=16$ and 18, respectively) the range of $F$ scores was twice as great as the range of $M$ scores. For Groups 2 and $4(n=21$ and 17 , respectively) the range of $M$ scores was twice the range of $F$ scores. See Table 1 for actual levels of each factor. The instructions for Groups 1 and 2 indicated that more emphasis should be put on the final than on the midterm but both $F$ and $M$ scores should be considered in assessing the given student's performance. No specific weighting was advocated. The instructions for Groups 3 and 4 specified that the final should be weighted twice as much as the midterm in rating students. Group descriptions are summarized in the left-hand columns of Table 2.

\section{Subjects and procedure}

Students from introductory psychology classes at the University of lowa were tested in groups of 8 to 12 . They were told that their task was to put themselves in the role of a college professor who has to compare and rate students in his class on the basis of midterm exam and final exam scores. Each hypothetical student's performance was summarized on a different page of a booklet. Two booklets were presented to each subject, one for each replication. A $20-\mathrm{cm}$ line labeled very good at one end and very bad at the other end was drawn on each page. Subjects were told to rate each student's performance by placing a slash mark somewhere across this line; the better the performance seemed, the closer to the very good end should be the mark; the worse the performance seemed, the closer to the very bad end should be the mark. Responses were recorded to the nearest centimeter and form a scale of 0 to 20 with higher numbers representing more favorable ratings. A line-mark

Table 1

Mean Ratings

\begin{tabular}{|c|c|c|c|c|c|c|c|}
\hline \multirow{2}{*}{$\begin{array}{c}\text { Final } \\
\text { Exam } \\
\text { Score (F) } \\
\end{array}$} & \multicolumn{5}{|c|}{ Group 1: Midterm Exam Score (M) } & \multirow[b]{2}{*}{ Mean } & \multirow[b]{2}{*}{ Final Only } \\
\hline & 59 & 64 & 69 & 74 & 79 & & \\
\hline $\begin{array}{r}87 \\
77 \\
67 \\
57 \\
47 \\
\text { Mean } \\
\text { Midterm Only } \\
\end{array}$ & $\begin{array}{r}13.59 \\
11.81 \\
10.19 \\
8.69 \\
7.66 \\
10.39 \\
8.63 \\
\end{array}$ & $\begin{array}{r}13.97 \\
12.03 \\
10.50 \\
9.69 \\
8.25 \\
10.89 \\
9.22 \\
\end{array}$ & $\begin{array}{r}14.03 \\
12.09 \\
10.88 \\
9.72 \\
8.31 \\
11.01 \\
9.75 \\
\end{array}$ & $\begin{array}{r}14.91 \\
13.22 \\
11.50 \\
10.53 \\
8.63 \\
11.76 \\
11.50\end{array}$ & $\begin{array}{r}15.41 \\
13.15 \\
11.72 \\
10.19 \\
9.28 \\
11.95 \\
11.10 \\
\end{array}$ & $\begin{array}{r}14.38 \\
12.46 \\
10.96 \\
9.76 \\
8.43 \\
11.20 \\
10.04 \\
\end{array}$ & $\begin{array}{r}15.28 \\
12.28 \\
10.38 \\
8.50 \\
5.57 \\
10.40\end{array}$ \\
\hline $\begin{array}{l}\text { Final } \\
\text { Exam }\end{array}$ & \multicolumn{5}{|c|}{ Group 2: Midterm Exam Score (M) } & & \\
\hline Score (F) & 47 & 57 & 67 & 77 & 87 & Mean & Final Only \\
\hline $\begin{array}{c}79 \\
74 \\
69 \\
64 \\
59 \\
\text { Mean } \\
\text { Midterm Only } \\
\end{array}$ & $\begin{array}{r}10.93 \\
10.62 \\
9.60 \\
9.74 \\
8.14 \\
9.81 \\
5.50 \\
\end{array}$ & $\begin{array}{r}11.88 \\
11.02 \\
10.19 \\
9.81 \\
8.79 \\
10.34 \\
8.24 \\
\end{array}$ & $\begin{array}{r}12.24 \\
11.79 \\
10.81 \\
10.50 \\
9.74 \\
11.01 \\
9.83 \\
\end{array}$ & $\begin{array}{l}13.60 \\
12.36 \\
11.71 \\
11.14 \\
10.64 \\
11.89 \\
11.55 \\
\end{array}$ & $\begin{array}{l}14.62 \\
14.24 \\
12.69 \\
12.57 \\
11.43 \\
13.11 \\
13.38 \\
\end{array}$ & $\begin{array}{r}12.65 \\
12.01 \\
11.00 \\
10.75 \\
9.75 \\
11.23 \\
9.70 \\
\end{array}$ & $\begin{array}{r}12.62 \\
11.74 \\
10.45 \\
10.05 \\
8.69 \\
10.71\end{array}$ \\
\hline $\begin{array}{l}\text { Final } \\
\text { Exam }\end{array}$ & \multicolumn{5}{|c|}{ Group 3: Midterm Exam Score (M) } & & \\
\hline Score (F) & 59 & 64 & 69 & 74 & 79 & Mean & Final Only \\
\hline $\begin{array}{c}87 \\
77 \\
67 \\
57 \\
47 \\
\text { Mean } \\
\text { Midterm Only } \\
\end{array}$ & $\begin{array}{r}13.64 \\
11.67 \\
10.36 \\
8.67 \\
7.08 \\
10.28 \\
7.81 \\
\end{array}$ & $\begin{array}{r}14.64 \\
12.14 \\
10.33 \\
9.19 \\
7.56 \\
10.77 \\
8.78 \\
\end{array}$ & $\begin{array}{r}14.39 \\
11.89 \\
10.25 \\
9.36 \\
8.06 \\
10.79 \\
9.17 \\
\end{array}$ & $\begin{array}{r}14.56 \\
12.78 \\
10.86 \\
9.83 \\
7.78 \\
11.16 \\
10.83 \\
\end{array}$ & $\begin{array}{r}15.31 \\
12.69 \\
11.00 \\
9.47 \\
8.61 \\
11.42 \\
11.17 \\
\end{array}$ & $\begin{array}{r}14.51 \\
12.23 \\
10.56 \\
9.30 \\
7.82 \\
10.88 \\
9.55 \\
\end{array}$ & $\begin{array}{r}14.92 \\
12.11 \\
10.56 \\
8.33 \\
5.75 \\
10.33\end{array}$ \\
\hline $\begin{array}{l}\text { Final } \\
\text { Exam }\end{array}$ & \multicolumn{5}{|c|}{ Group 4: Midterm Exam Score (M) } & & \\
\hline$\underline{\text { Score }(F)}$ & 47 & 57 & 67 & 77 & 87 & Mean & Final Only \\
\hline $\begin{array}{c}79 \\
74 \\
69 \\
64 \\
59 \\
\text { Mean } \\
\text { Midterm Only } \\
\end{array}$ & $\begin{array}{r}12.21 \\
11.76 \\
10.62 \\
10.76 \\
9.21 \\
10.91 \\
5.62 \\
\end{array}$ & $\begin{array}{r}12.65 \\
12.24 \\
10.68 \\
10.79 \\
9.56 \\
11.18 \\
7.59 \\
\end{array}$ & $\begin{array}{r}13.15 \\
12.38 \\
11.50 \\
10.88 \\
10.62 \\
11.71 \\
8.91 \\
\end{array}$ & $\begin{array}{l}14.03 \\
13.29 \\
12.26 \\
11.76 \\
10.97 \\
12.46 \\
10.97 \\
\end{array}$ & $\begin{array}{l}14.91 \\
14.27 \\
12.68 \\
12.44 \\
11.79 \\
13.22 \\
12.09 \\
\end{array}$ & $\begin{array}{r}13.39 \\
12.79 \\
11.55 \\
11.33 \\
10.43 \\
11.90 \\
9.04 \\
\end{array}$ & $\begin{array}{r}12.15 \\
11.65 \\
10.68 \\
10.18 \\
8.82 \\
10.70\end{array}$ \\
\hline
\end{tabular}


response scale such as this has been validated in previous studies (e.g., Levin, 1975).

Subjects were told that in most cases a student's performance would be described by both a midterm and a final exam score, but that in some cases only one piece of information would be presented, representing a student who missed an exam. In the former cases, they were told to base their ratings on both pieces of information, and in the latter cases they were told to base their ratings on only the single score. While ratings based on combinations of scores were of primary interest, comparisons between these ratings and ratings based on single scores were included to help discriminate between alternative models of the information integration process.

Before rating each individual student's performance, subjects were allowed to look through the pages of their first booklet to examine the relative performance of each student. They were instructed to then rate the performance of each student in relation to the performance of other students. Ratings were self-paced but subjects were not allowed to go back and change earlier responses. The average time for completing the task was about $25 \mathrm{~min}$.

\section{RESULTS}

Mean rating responses are summarized in Table 1. For each group, Table 1 gives the mean rating for each combination of $\mathrm{F}$ and $\mathrm{M}$ scores, the mean for each row and column of the factorial design, and the mean rating for each level of $F$ and $M$ on trials where only one score was presented. Results are combined for both replications of the design because the replications factor did not have a significant effect for any group and was involved in only one minor interaction effect.

Results were analyzed in the following sequence: (1) Ratings based on combinations of $F$ and $M$ scores were subjected to analysis of variance to determine the appropriateness of an additive model in describing how subjects combine the two pieces of information. (2) Ratings based on single scores were compared to ratings based on combinations to discriminate between an adding model and an averaging model. (3) The model was used to obtain estimates of the weights assigned to $F$ and M scores. (4) Weight estimates were compared across experimental groups to see if they are invariant over changes in scale value ranges. (5) Weights derived for groups instructed to weight the final twice as much as the midterm were compared to actual $2: 1$ weights.

\section{The form of the integration model}

Ratings of combinations of $F$ and $M$ scores were analyzed for each group separately. The main effect of each type of score was large and highly significant for each group. (F-ratios for main effects ranged from about 15 to 180 , with MSE ranging from about 2 to 9.) The Midterm by Final interaction did not reach statistical significance in Groups 1 and 4 . The interaction term was significant at the .05 (but not the .01 ) level for Groups 2 and $3[\mathrm{~F}(16 / 320)=1.99$. MSE $=1.23$ and $F(16 / 272)=1.77$. MSE $=1.48$, respectively]. The presence of interaction effects generally discredits an additive model and/or indicates a nonlinearity of the response scale. In the present case, scores tended to be more spread out for the extreme values of each stimulus dimension than for the middle values. This could mean that the unit of the subjective scale was larger for middle values than for extreme values. However, the interaction effect was very small for each group and never contributed more than .004 of the variance contributed by the main effects. In other words, the departure from additivity was extremely slight.

An additive model thus appears to provide a reasonably good description of the results for each group. The general additive model can take one of two specific forms - an adding model or an averaging model. These models can be differentially tested when information sets vary in size (Anderson, 1965; Levin, 1974a). In the present case, this is accomplished by comparing the marginal means of the factorial design for final-midterm combinations with the mean ratings for trials in which only an $\mathrm{F}$ or $\mathrm{M}$ score was given. For each group the relevant comparisons are between the two bottom rows of means and between the two right-hand columns of means in Table 1. If an adding model holds, then the addition of a given $M$ score to each value of $F$ should increment each rating by the same amount. The range of mean values for F-only scores should then be the same as the range of marginal row means. Similarly, the adding model implies that the range of mean values for M-only scores will be the same as the range of marginal column means. If, on the other hand, an averaging model holds, the addition of a given $M$ score to each value of $F$ could increase or decrease the ratings. depending on whether the value of $\mathrm{M}$ was more or less extreme than the value of $F$. The range of mean-values for F-only scores would then be greater than the range of marginal row means, and, similarly, the range of mean values for $\mathrm{M}$-only scores would be greater than the range of marginal column means.

Consistent with an averaging model, all groups had a smaller range for the marginal means of the factorial design than for the single-score ratings for both rows and columns. (The fact that single-score ratings were, on the average, lower in magnitude than ratings based on both $F$ and $M$ scores probably indicates that subjects discredited a student who missed an exam, particularly if it was the final.) Ratings (R) of hypothetical students based on both $F$ and $M$ scores can be described by a model of the following form:

$$
R=\frac{w_{0} I_{o}+w_{F}^{F}+w_{M}^{M}}{w_{0}+w_{F}+w_{M}}
$$

where $w_{0}$ and $I_{0}$ represent, respectively, the weight and value of the subject's initial expectancy or response disposition; $w_{F}$ and $w_{M}$ represent the weight assigned to the final and the midterm, respectively. Dividing by the sum of the weights makes this an averaging model. The 


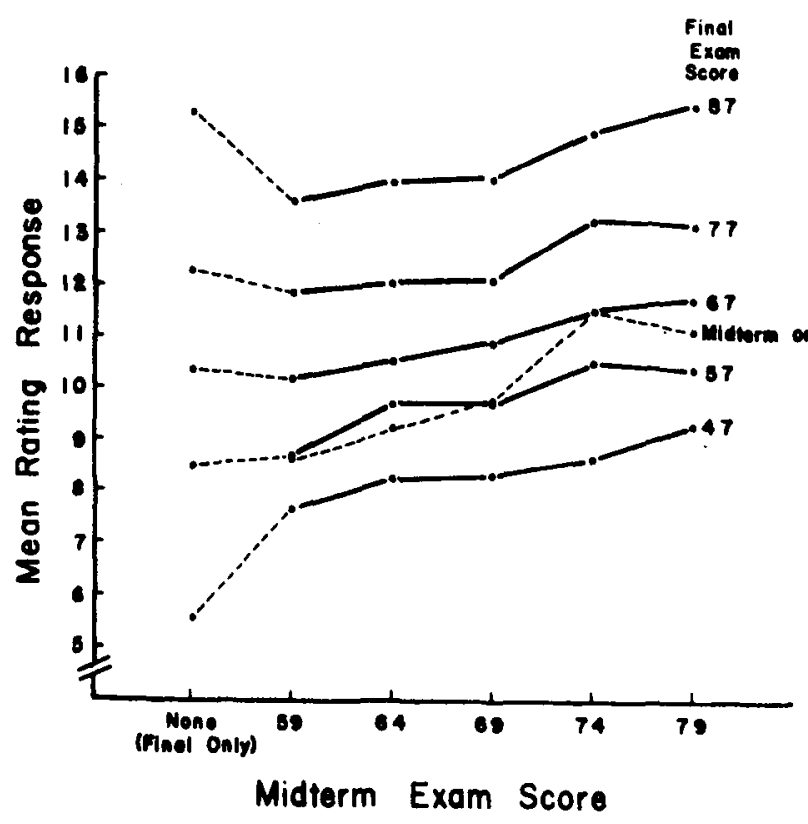

Figure 1. Mean rating responses for Group 1. Solid lines represent ratings based on both $F$ and $M$ scores. Dotted lines represent ratings based on single scores.

inclusion of an initial expectancy has been shown to be useful in explaining a number of phenomena associated with averaging mechanisms (Anderson, 1974). Since the weights are not assumed to vary as a function of the actual levels of $F$ and $M$, this model is called an equal-weight averaging model. Since the mean rating is greater than 10 for each group, it can be assumed that the initial response disposition $\mathrm{I}_{0}$ tends to be biased in the positive direction. This is consistent with earlier work (Levin, 1975).

Figure 1 illustrates the tests described in this section. Mean rating responses for midterm-final combinations are plotted for Group 1. The near-parallelism of the solid lines supports an additive model. The dotted lines represent judgments based on only one score and show that they have more extreme ranges than the rows and columns of the factorial design.

\section{Analy sis of weighting factors}

The relative influence of $F$ and $M$ scores can be assessed by comparing the range of marginal means for row and column variables of Table 1 . In an equal-weight averaging model such as described in Equation 1, the ratio of ranges of the marginal means equals the ratio of weights times the ratio of the scale value ranges of the two variables. This then formalizes the confounding described earlier where the relative influence of two variables is a joint function of their weights and the ranges over which they are varied. In this case, however, the confounding can be resolved.

Previous research in analogous tasks (e.g., Levin, 1975) showed that numbers themselves can be taken as interval scale estimates of their subjective scale values. The ratio of scale value ranges can thus be gotten directly from the actual ranges of values used. The appropriate ratios (r) are 2:1 for Groups 1 and 3, and $1: 2$ for Groups 2 and 4 . These ratios can be used to obtain weight estimates for each group.

In practice, rather than using the range of marginal means, which involves only two row or column means, an analogous but more stable measure of the relative influence of $F$ and $M$ scores was used - the slope of the best-fitting straight line for each array of marginal mean values. (Another measure yielding similar results is the square root of the mean square for each variable. Such a measure has been used by Norman, Reference Note 1.) The equation for obtaining weights can now be expressed as follows:

$$
\left(w_{F} / w_{M}\right)=(s \bar{F} / s \bar{M}) \times r^{-1}
$$

where $s_{F}$ and $s_{M}$ represent the slope of the best-fitting straight line for $F$ and $M$ marginal values, respectively, $r=2$ for Groups 1 and 3, and $r=1 / 2$ for Groups 2 and 4 . For convenience, the weights $w_{F}$ and $w_{M}$ were normalized to sum to 1 . The weights obtained from Equation 2 are given in Table 2 .

It can be seen in Table 2 that the weight estimates obtained for Groups 1 and 2 are nearly identical, and the weights for Groups 3 and 4 are also close. To compare these values statistically, $w_{F}$ was estimated for each individual subject and $t$ tests were used to compare the mean value of $w_{F}$ for each pair of groups. (It should be noted that the mean value of the weight for individual subjects in a group does not necessarily equal the weight obtained from the grouped data, but in the present case these values were close.) Neither the difference between Groups 1 and 2 nor the difference between Groups 3 and 4 approached statistical significance, $[\mathrm{t}(35)=0.68$, $\mathrm{SE}=.038$. and $\mathrm{t}(33)=0.50, \mathrm{SE}=0.42$, respectively $]$.

Table 2

Weight Estimates

\begin{tabular}{|c|c|c|c|c|c|c|c|c|c|c|}
\hline \multirow[b]{2}{*}{ Group } & \multirow[b]{2}{*}{$\begin{array}{l}\text { Weighting } \\
\text { Instructions }\end{array}$} & \multicolumn{3}{|c|}{ Score Ranges } & \multicolumn{3}{|c|}{ Slope of Marginal Mean Arrays } & \multicolumn{3}{|c|}{ Weights } \\
\hline & & $\begin{array}{c}\text { Row } \\
\text { (F) }\end{array}$ & $\begin{array}{l}\text { Column } \\
\text { (M) }\end{array}$ & Ratio & $\begin{array}{c}\text { Row } \\
\text { (F) }\end{array}$ & $\begin{array}{l}\text { Column } \\
\text { (M) }\end{array}$ & Ratio & $\begin{array}{l}\text { Final } \\
\left(w_{F}\right)\end{array}$ & $\begin{array}{c}\text { Midterm } \\
\left(\mathrm{w}_{\mathbf{M}}\right)\end{array}$ & Ratio \\
\hline 1 & $\mathbf{w}_{\mathbf{F}}>\mathbf{w}_{\mathbf{M}}$ & 40 & 20 & 2 & 1.460 & .399 & 3.66 & .65 & .35 & 1.83 \\
\hline 2 & $w_{F}>w_{M}$ & 20 & 40 & $1 / 2$ & .706 & .815 & .87 & .63 & .37 & 1.74 \\
\hline 3 & $w_{F}=2 w_{M}$ & 40 & 20 & 2 & 1.629 & .269 & 6.06 & .75 & .25 & 3.03 \\
\hline 4 & $w_{F}=2 w_{M}$ & 20 & 40 & $1 / 2$ & .738 & .590 & 1.25 & .71 & .29 & 2.50 \\
\hline
\end{tabular}


Since Groups 1 and 2 and Groups 3 and 4 differed greatly in the relative ranges of the factors manipulated, these results support the contention that weights are invariant over scale value ranges.

Groups 3 and 4 were instructed to weight the final exam twice as much as the midterm exam in rating the performance of students. When weight estimates were combined for these two groups, these groups were found to have weighted the final exam significantly more than twice as much as the midterm, $[\mathrm{t}(34)=2.77, \mathrm{SE}=.020$, $\mathrm{p}<.01]$. In fact, the weights for Groups 1 and 2, who were not told to assign a specific weighting, were closer to $2: 1$ than were the weights for Groups 3 and 4 .

\section{DISCUSSION}

The major finding of this study is that the relative weights of the two stimulus dimensions were invariant across variations in the range of values of the dimensions. This provides direct support for the basic assumption of Anderson's (1974) information integration theory that each piece of information in an integration task has two separate components, a weight and a scale value, where the weight parameter reflects the degree of psychological importance of an informational stimulus. This assumption has recently been debated by Schönemann, Cafferty, and Rotton (1973) and Anderson (1973). Schönemann et al. claim that weights are "empirically empty parameters," whereas Anderson asserts that both weight and scale value are required to characterize an informational stimulus. Invariance of the weight parameter in the present study tends to support the conceptualization of weight as a measure of psychological importance.

The present finding that weights were invariant across changes in stimulus range does not imply that this will always be the case. Several studies of psychophysical averaging have shown that more intense stimuli have greater weight as well as greater scale value (Levin, Norman, \& Dolezal, 1973; Parducci, Thaler, \& Anderson, 1968) and studies of personality impression formation have indicated that unfavorable information carries more weight than favorable information (Levin, Wall, Dolezal, \& Norman, 1973). In these instances an equal-weight averaging model would not be supported. Thus the present method for testing weight invariance has restricted application. It is meant to apply to tasks where an equal-weight averaging model provides a good description of the judgmental process and where units of the subjective scale are constant across and between stimulus dimensions. This latter assumption was not tested directly in the present study but the obtained weight invariance provides indirect support for the assumption. Norman (Reference Note 1) has devised a method for separating weights and scale values which does not rely on the assumption of equal units across stimulus dimensions. Norman's method includes variation of the number of stimulus factors to be combined and is therefore analogous to the present use of single-stimulus ratings in conjunction with ratings based on stimulus combinations. Parameter invariance has not yet been tested with this method. The demonstration of weight invariance in the present study is important in spite of the restricted boundary conditions.

An additional finding was that subjects could not accurately produce a designated set of weights. Other studies (Levin, 1973; Norman, 1974) have shown that feedback and instructions can effect directional changes in the weights used by subjects, but the mathematical matching of designated weights is apparently more difficult. The actual weights used, however, could be estimated from a descriptive model of information integration. This points to the advantages of descriptive over normative models - see also Shanteau (1972) and Levin (1975) - and underscores the usefulness of formal models to determine the relative degree of psychological importance of each variable in a judgmental task.

\section{REFERENCE NOTE}

1. Norman, K. L. A solution for weights and scale values in functional measurement. Manuscript submitted for publication, 1975 .

\section{REFERENCES}

Anderson, N. H. Averaging versus adding as a stimulus-combination rule in impression formation. Journal of Experimental Psychology, 1965, 70, 394-400.

Anderson, N. H. Comments on the articles of Hodges and of Schönemann, Cafferty, and Rotton. Psychological Review, $1973,80,88-92$.

Anderson, N. H. Information integration theory: A brief survey. In D. H. Krantz, R. C. Atkinson, R. D. Luce, \& P. Suppes (Eds.), Contemporary developments in mathematical psychology. Vol. 2. San Francisco: Freeman, 1974.

Dooling, D. J., \& Danks, J. H. Going beyond tests of significance: Is psychology ready? Bulletin of the Psychonomic Society, 1975, 5, 15-17.

Levin, I. P. Learning effects in information integration: Manipulation of cue validity in an impression formation task. Memory \& Cognition, 1973, 1, 235-240.

Levin, I. P. Averaging processes in ratings and choices based on numerical information. Memory \& Cognition, 1974, 2, 786-790. a.

Levin, I, P. Averaging processes and intuitive statistical judgments. Organizational Behavior and Human Performance, $1974,12,83-91$, b.

Levin, I. P. Information integration in numerical judgments and decision processes. Journal of Experimental Psychology: General, 1975, 104, 39-53.

Levin, I. P., Norman, K. L., \& Dolezal, J. M. Response scale effects and integration processes in the averaging of motor movements. Journal of Motor Beh avior, 1973, 5, 1-7.

Levin, I. P., Wall, L. L., Dolezal, J. M., \& Norman, K. L. Differential weighting of positive and negative traits in impression formation as a function of prior exposure. Joumal of Experimental Psy chology, 1973, 97, 114-115.

Norman, K. L. Dynamic processes in stimulus integration theory: The effects of feedback on the averaging of motor movements. Journal of Experimental Psychology, 1974, 102, 399-408.

Parducci, A., Thaler, L., \& Anderson, N. H. Stimulus averaging and the context for judgment. Perception \& Psychophysics, $1968,3,145-150$.

Schönemann, P. H.. Cafferty, T., \& Rotton, J. A note on additive functional measurement. Psychological Review, 1973, 80, 85-87.

Shanteau, J. Descriptive versus normative models of sequential inference judgment. Joumal of Experimental Psychology, $1972,93,63-68$

(Received for publication January 27, 1975. (Revision received April 17, 1975.) 